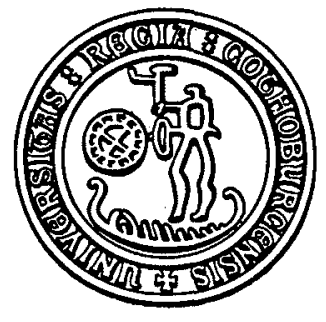

Research Report

Department of Statistics

Göteborg University

Sweden

\title{
Trend-free repeated \\ measurement designs
}

Kasra Afsarinejad

Research Report 1999:9

ISSN 0349-8034

Mailing address:

Dept of Statistics

P.O. Box 660

Fax

Nat: 031-773 1274

Int: +4631773 1274

Phone

Nat: 031-773 1000

SE 40530 Göteborg

Sweden 


\title{
TREND-FREE REPEATED MEASUREMENT DESIGNS
}

\author{
K. Afsarinejad \\ Department of Statistics, Göteborg University, \\ Box 660, 40530 Göteborg, Sweden
}

Key Words and Phrases: changeover; crossover; optimal design; orthogonal polynomial; residual effect; trend-free.

\begin{abstract}
The existence and non-existence of trend-free repeated measurement designs are investigated. Two families of efficient / optimal trend-free repeated measurement designs have been identified and methods of construction of these designs are presented.
\end{abstract}

\section{INTRODUCTION}

In many industrial, medical and agricultural experiments, treatments are applied to each experimental unit sequentially in time or space. There is a possibility that a systematic effect, or trend, influences the observations in addition to the experimental unit, treatment and residual effects. This type of effects should be taken into account both when the experiment is planned and when the results are analysed. One way to account for the presence of trends is to use the analysis of covariance, treating trend values as covariates. However, one may use suitable designs in the presence of trends to avoid the complications of analysis of covariance and increase design efficiencies.

Some studies in this direction have been made by Atkinson and Donev (1996), Box (1952), Box and Hay (1953), Bailey, Cheng and Kipnis (1992), Bradley and Yeh (1985), Bradley and Odeh (1988), Chai and Majumdar (1993), Cheng (1985), Cheng and Jacroux (1988), Coster (1993), Coster and Cheng (1988), Cox $(1951,1952,1958)$, Daniel (1976, chapter 15), Daniel and wilcoxon (1966), Githinji and Jacroux (1998), Hill (1960), Jaroux (1993, 1994), Jacroux and Ray (1990), Joiner and Campbell (1976), Lin and Dean (1991), Mukerjee and Sengupta (1994), Ogilvie(1963), Philips (1964,1968a,1968b), Prescott (1981), Stufken (1988), Taylor (1967), Yeh and Bradley (1983), Yeh, Bradley and Notz (1985), Whittinghill (1989) and Wilkie (1987).

In the present paper, we consider two families of repeated measurement designs (RMDs) and show that efficient/optimal trend-free RMDs exist.

In Section 2 we give the notation and basic definitions for RMDs. The model and conditions for trend-free RMDs are discussed in Section3. Finally, Section 4 contains the main results. 


\section{NOTATION AND DEFINITIONS}

In an RMD there are $t$ treatments, $n$ experimental units and $\mathrm{p}$ periods. Each experimental unit receives one treatment during each period. Thus a RMD denoted by

$\mathrm{RMD}(\mathrm{t}, \mathrm{n}, \mathrm{p})$ is a $\mathrm{p} \times \mathrm{n}$ array with treatments as entries, rows representing periods and columns as experimental units. The set of all such arbitrary RMDs is designated by $\Omega_{\mathrm{t}, \mathrm{n}, \mathrm{p}}$. Let us formally define some useful concepts.

Definition 2.1. A design $\mathrm{d} \in \Omega_{\mathrm{t}, \mathrm{n}, \mathrm{p}}$ is said to be uniform on periods if each treatment is administered $\lambda_{1}$ times during each period.

Definition 2.2. A design $\mathrm{d} \in \Omega_{\mathrm{t}, \mathrm{n}, \mathrm{p}}$ is said to be uniform on experimental units if each treatment is administered to each experimental unit $\lambda_{2}$ times.

Definition 2.3. A design $\mathrm{d} \in \Omega_{\mathrm{t}, \mathrm{n}, \mathrm{p}}$ is said to be uniform if it is uniform on periods and experimental units simultaneously.

Definition 2.4. A design $d \in \Omega_{t, n, p}$ is said to be balanced for residuals if in the pxn array $d$ each treatment is preceded by each other treatment (not including itself) $\lambda$ times.

Definition 2.5. A design $\mathrm{d} \in \Omega_{\mathrm{t}, \mathrm{n}, \mathrm{p}}$ is said to be strongly balanced for residuals if each treatment is preceded by all the other treatments (including itself) $\lambda$ times.

The necessary conditions for the respective RMDs listed above are:

$\mathrm{n}=\lambda_{1} \mathrm{t}, \mathrm{p}=\lambda_{2} \mathrm{t}, \mathrm{n}(\mathrm{p}-1)=\lambda \mathrm{t}(\mathrm{t}-1)$ and $\mathrm{n}=\lambda_{1} \mathrm{t}, \mathrm{p}=\lambda_{2} \mathrm{t}, \mathrm{n}(\mathrm{p}-1)=\lambda \mathrm{t}^{2}$, respectively.

Definition 2.6. In case $\mathrm{p}<\mathrm{t}$ we call a design $\mathrm{d} \in \Omega_{\mathrm{t}, \mathrm{n}, \mathrm{p}}$ balanced if it is uniform on periods, balanced for residuals and each experimental unit is administered distinct treatments.

Definition 2.7. In case $\mathrm{p}<\mathrm{t}$ we call a design $\mathrm{d} \in \Omega_{\mathrm{t}, \mathrm{n}, \mathrm{p}}$ strongly balanced if it is uniform on periods and strongly balanced for residuals.

The necessary conditions for the respective RMDs listed above are:

$\mathrm{n}=\lambda_{1} \mathrm{t}, \mathrm{n}(\mathrm{p}-1)=\lambda \mathrm{t}(\mathrm{t}-1)$ and $\mathrm{n}=\lambda_{1} \mathrm{t}, \mathrm{n}(\mathrm{p}-1)=\lambda \mathrm{t}^{2}$ respectively.

Definition 2.8. A design $d \in \Omega_{\mathrm{t}, \mathrm{n}, \mathrm{p}}$ is called circular balanced if the collection of ordered pair $(\mathrm{d}(\mathrm{i}, \mathrm{j}), \mathrm{d}(\mathrm{i}+1, \mathrm{j})), 1 \leq \mathrm{i} \leq \mathrm{p}, 1 \leq \mathrm{j} \leq \mathrm{n}$ contains each pair of distinct treatments $\lambda$ times.

Definition 2.9. A design $\mathrm{d} \in \Omega_{\mathrm{t}, \mathrm{n}, \mathrm{p}}$ is called circular strongly balanced if the collection of ordered pairs $(d(i, j), d(i+1, j)), 1 \leq i \leq p, 1 \leq j \leq n$ contains each ordered pair of treatments (distinct or not) $\lambda$ times.

In the last two definitions, when $i=p, i+1=1$, since we count the pairs $(d(p, j), d(1, j))$ as well.

The necessary conditions for the respective designs listed above are:

$\mathrm{np}=\lambda \mathrm{t}(\mathrm{t}-1)$ and $\mathrm{np}=\lambda \mathrm{t}^{2}$ respectively.

\section{Model and conditions for trend-free RMDS}

We assume that, within each experimental unit there is a common polynomial trend of order $\mathrm{k}$ on the p periods, which can be expressed by the orthogonal polynomials $\phi_{\alpha}(l), 1 \leq \alpha$ $\leq \mathrm{k}$, on $l=1, \ldots, \mathrm{p}$, where $\phi_{\alpha}$ is a polynomial of degree $\alpha$. The polynomials $\phi_{1}(l), \ldots, \phi_{\mathrm{k}}(l)$ satisfy 


$$
\sum_{l=1}^{p} \phi_{\alpha}(l)=0, \quad \quad \sum_{l=1}^{p} \phi_{\alpha}(l) \phi_{\alpha^{\prime}}(l)=\delta_{\alpha \alpha^{\prime}},
$$

Where $\delta_{\alpha \alpha^{\prime}}$ denotes the Kronecker delta, $\alpha, \alpha^{\prime}=1, \ldots, \mathrm{k}$.

Let $\mathbf{X}_{\beta}=\left[\mathbf{I}_{\mathrm{n}} \otimes \mathbf{1}_{\mathrm{p}}\right], \mathbf{X}_{\theta}=\left[\mathbf{1}_{\mathrm{n}} \otimes \Phi_{\mathrm{k}}\right]$, where $\mathbf{1}_{\mathrm{n}}$ is a $\mathrm{n} \times 1$ vector of unit elements, $\mathbf{I}_{\mathrm{n}}$ is a $\mathrm{n} \times \mathrm{n}$ identity matrix, $\otimes$ denotes a Kronecker product and $\Phi_{\mathrm{k}}$ is a $\mathrm{p} \times \mathrm{k}$ matrix with elements $\phi_{\alpha}(l)$ in row $l$ and column $\alpha, l=1, \ldots, \mathrm{p}, \alpha=1, \ldots, \mathrm{k}$. Then listing the response variables in the vector $\mathrm{Y}$ in order of period position within successive experimental units, the standard model for a RMD with trend is:

$$
\mathrm{E}(\mathrm{Y})=\mathrm{X}_{\mu} \mu+\mathrm{X}_{\tau} \tau+\mathrm{X}_{\rho} \rho+\mathrm{X}_{\beta} \beta+\mathrm{X}_{\theta} \sigma
$$

Where $\mu$ is a constant, $X_{\mu}=1_{\mathrm{np}}, \tau$ is the $\mathrm{t} \times 1$ vector of treatment parameters, $X_{\imath}=$ $\left[\Delta_{1}, \Delta_{2}, \ldots, \Delta_{n}\right]$ where for $\mathrm{i}=1, \ldots, \mathrm{n}, \Delta_{\mathrm{n}}$ is a pxt matrix whose $(\mathrm{k}, \mathrm{j})$ th element is unity if treatment $\mathrm{j}$ is applied in period $\mathrm{k}$, and zero otherwise, $\rho$ is the tx 1 vector of residuals, $X_{\rho}$ $=\left[\Pi_{1}, \Pi_{2}, \ldots, \Pi_{n}\right]$ where for $i=1, \ldots, n, \Pi_{i}^{\prime}$ is a pxt matrix whose $(k, j)$ element is unity if treatment $\mathrm{k}$ is applied to the period preceding the $\mathrm{j}$-th period, and zero otherwise.

Now we are in a position to define trend-free RMDs.

Definition 3.1 An RMD is trend-free with respect to treatments if $X_{\tau}^{\prime} X_{\theta}=0$.

Definition 3.2 An RMD is trend-free with respect to residuals if $X_{\rho}^{\prime} X_{\theta}=0$.

Definition 3.3. An RMD is trend-free if it is trend-free with respect to both treatments and residuals.

For design d let $\mathrm{u}_{d i l}$ and $\mathrm{v}_{\mathrm{djl}}$ denote the number of times treatment $\mathrm{i}$ appears in period $l$ and the number of times residual $\mathrm{j}$ appears in period $l$, respectively. A design is linear trendfree with respect to treatments if and only if

$$
\sum_{i=1}^{p} u_{d i l} \phi_{1}(l)=0, \quad \mathrm{i}=1, \ldots, \mathrm{t} .
$$

A design is linear trend-free with respect to residuals if and only if

$$
\sum_{j=1}^{p} v_{d j l} \phi_{1}(l)=0, \quad \mathrm{j}=1, \ldots, \mathrm{t} .
$$

The polynomial, $\phi_{1}(l)$ satisfies $\phi_{1}(l)=-\phi_{1}(\mathrm{p}-l+1)$. For $\mathrm{k}$ odd $\phi_{1}((\mathrm{k}+1) / 2)=0$. Clearly (3.2) and (3.3) are true whenever

$$
\mathrm{u}_{\mathrm{dil}}=\mathrm{u}_{\mathrm{di}(\mathrm{p}-l+1)} \text { and } \mathrm{v}_{\mathrm{djl}}=\mathrm{v}_{\mathrm{dj}(\mathrm{p}-l+1)} \quad l=1, \ldots,[(\mathrm{p}+1) / 2], \mathrm{i}, \mathrm{j}=1, \ldots, \mathrm{t},
$$

where [ ] denotes the largest integer less than or equal to $(\mathrm{p}+1) / 2$.

Conditions (3.2) and (3.3) do not imply condition (3.4). Condition (3.4) is necessary and sufficient for a design to be odd-degree trend-free [Lin and Dean (1991), corollary 2.1.2].

The following theorem is a modification to Corollary (2.1.1) of Lin and Dean (1991). Theorem 3.1. A design $d \in R M D(t, n, p)$ which is trend-free with respect to treatments (residuals) for a (p-1)th order polynomial trend exists if and only if it is possible to arrange the treatments (residuals) so that each treatment (residual) occurs $\mathrm{p}^{-1} \mathrm{r}$ times in each periods. 


\section{Main results.}

In this section we are going to obtain some classes of trend-free RMDs and RMDs which are trend-free with respect to treatments.

First we shall consider RMDs which are trend-free with respect to treatments.

Theorem 4.1. All the balanced or strongly balanced RMDs which are uniform on periods are also trend-free with respect to treatments.

Proof . Each treatment appears the same number of times in each period so by Theorem (3.1) they are trend-free with respect to treatments.

There are two families of balanced RMDs.

Family 1. $p=t$. Balanced $R M D(t, t, t)$ exists for all even $t$. A method of construction is presented below.

Construction 4.1. Construct a txt table in which columns represents experimental units and rows represent periods. Number the $t$ experimental units successively from 1 to $t$. Assign integer 1 to $t$ to the $t$ cells in the first column by entering successive numbers in every other cell from top to bottom beginning with the first. Reverse the direction, going from bottom to top, once the end of a column has been reached, making sure that the return starts from the $\mathrm{t}$-th cell in the column. Finally, complete each row in a cyclic manner.

Example 4.1. Let $t=4$. Then the above procedure produces the following design

$\begin{array}{llll}1 & 2 & 3 & 4 \\ 4 & 1 & 2 & 3 \\ 2 & 3 & 4 & 1 \\ 3 & 4 & 1 & 2\end{array}$

If $t$ is odd then balanced $\operatorname{RMD}(t, t, t)$ does not exist for $t=3,5,7$. Balanced $\operatorname{RMD}(t, t, t)$ exists for $t=9,15,21,27,39,55$ and 57 . The existence and non-existence of balanced $\operatorname{RMD}(t, t, t)$ is not known. One can trivially construct balanced $\operatorname{RMD}(t, 2 t, t)$ for all odd $t$. An easily remembered method is given next.

Construction 4.2. Construct a design for $t$ experimental units analogous to method 3.1 outlined for t even. Similarly, construct for the remaining $t$ experimental units a design by letting the first column be the reverse of the first column of the design constructed for the initial $t$ units. The following example illustrates the above method.

Example 4.2. Let $t=5$. Then the above procedure produces the following design.

$\begin{array}{llllllllll}1 & 2 & 3 & 4 & 5 & 3 & 4 & 5 & 1 & 2 \\ 5 & 1 & 2 & 3 & 4 & 4 & 5 & 1 & 2 & 3 \\ 2 & 3 & 4 & 5 & 1 & 2 & 3 & 4 & 5 & 1 \\ 4 & 5 & 1 & 2 & 3 & 5 & 1 & 2 & 3 & 4 \\ 3 & 4 & 5 & 1 & 2 & 1 & 2 & 3 & 4 & 5\end{array}$

Unfortunately these designs are not trend-free with respect to residuals. To get around this problem one can consider the class of circular balanced RMDs. In this class the structure of residuals and treatments is the same and therefore designs in this class are trend-free. 
One can introduce a preperiod ( period 0 ) giving all the periods residual effects. The period 0 would not be used in analysis. Circular RMDs exist whenever $t>2$. Sonnemann, quoted in Kunert (1985), gave the following construction method for a circular balanced uniform RMDs with a minimum number of experimental units whenever $t>2$ is an even integer.

Construction 4.3. Let $\mathrm{t}=2 \mathrm{k}$ and obtain the first set of $\mathrm{t}$ columns by developing the column $(2 \mathrm{k} 12 \mathrm{k}-122 \mathrm{k}-23 \ldots \mathrm{k}+1 \mathrm{k})^{\prime}$ and call it $\mathrm{D}_{0}$. Let $\pi=\left(\begin{array}{lll}1 & 2 \ldots \mathrm{k}-1 & 2 \mathrm{k}-1 \ldots \mathrm{k})\end{array}\right)$ and let $\mathrm{D}_{\mathrm{i}}=$ $\pi^{\mathrm{i}} \mathrm{D}_{0}, \mathrm{i}=1,2, \ldots, \mathrm{t}-2$. Then $\mathrm{D}_{0}, \mathrm{D}_{1}, \ldots, \mathrm{D}_{\mathrm{t}-2}$ can be juxtaposed to obtain the required RMD. Example 4.3. Let $\mathrm{k}=3, \mathrm{t}=6$; then $\pi=(12543)$ and

$\begin{array}{rr}612345 & 625134 \\ 123456 & 251346 \\ \mathrm{D}_{0}= & 561234 \\ 234561 & \mathrm{D}_{1}=462513 \\ 456123 & 513462 \\ 345612 & 346251 \\ & 134625 \\ 654213 & \\ 542136 & 643521 \\ \mathrm{D}_{2}= & 465421 \\ 421365 & \mathrm{D}_{3}=164352 \\ 136542 & 352164 \\ 213654 & 216435 \\ & \\ 631452 & 521643 \\ 314526 & \\ \mathrm{D}_{4}= & \\ 143145 & \\ 526314 & \\ 452631 & \end{array}$

Afsarinejad ( 1990), using disjoint directed Hamiltonian cycles, constructed circular balanced uniform RMDs with minimum number of experimental units whenever $t$ is an odd number.

Construction 4.4. Let $\mathrm{t}=2 \mathrm{k}+1$ and label the $\mathrm{t}$ treatments by $0,1,2, \ldots, 2 \mathrm{k}$. Then construct $2 \mathrm{k}$ columns as follows:

$$
\begin{aligned}
& C_{i}^{+}=(0 \mathrm{i} i+1 \mathrm{i}-1 \mathrm{i}+2 \mathrm{i}-2 \ldots \mathrm{i}+(\mathrm{k}-1) \mathrm{i}-(\mathrm{k}-1) \mathrm{i}+\mathrm{k})^{\prime} \\
& C_{i}^{-}=(0 \mathrm{i}+\mathrm{ki}-(\mathrm{k}-1) \mathrm{i}+(\mathrm{k}+1) \ldots \mathrm{i}-2 \mathrm{i}+2 \mathrm{i}-1 \mathrm{i}+1 \mathrm{i})^{\prime}
\end{aligned}
$$

for $1 \leq i \leq k$. All the elements except 0 are taken as the positive integers $1,2, \ldots, 2 \mathrm{k}$ (mod $2 \mathrm{k})$.

These $2 \mathrm{k}$ columns are initial columns of $2 \mathrm{k}$ squares, which can be developed in turn to obtain $2 \mathrm{k}$ squares. Juxtaposing these squares gives a circular balanced uniform RMD with a minimum number of experimental units.

Example 4.5. Let $\mathrm{t}=5$. Then the four columns are: 


$$
\begin{aligned}
& C_{1}^{+}=\left(\begin{array}{llll}
0 & 1 & 2 & 4
\end{array}\right)^{\prime} \\
& C_{2}^{+}=\left(\begin{array}{lllll}
0 & 2 & 3 & 1 & 4
\end{array}\right)^{\prime} \\
& C_{1}^{-}=\left(\begin{array}{llll}
0 & 3 & 4 & 2
\end{array}\right)^{\prime} \\
& C_{2}^{-}=\left(\begin{array}{lllll}
0 & 4 & 1 & 3 & 2
\end{array}\right)^{\prime}
\end{aligned}
$$

The required design is:

$$
\begin{aligned}
& 01243023140342104132 \\
& 12430231403421041320 \\
& 24301314024210313204 \\
& 43012140232103432041 \\
& 30124402311034220413
\end{aligned}
$$

Family 2. $\mathrm{p}<\mathrm{t}$.

Afsarinejad (1983) constructed all the possible balanced minimal RMDs and strongly balanced minimal RMDs. These designs are all trend-free with respect to treatments.

Construction 4.5. A balanced minimal RMD can be obtained by developing, in turn, mod $t$, each of the following $\lambda_{1}$ columns.

$$
\begin{aligned}
& \mathrm{c}_{1} \mathrm{c}_{\mathrm{p}} \ldots \mathrm{c}_{(\mathrm{i}-1) \mathrm{p}-(\mathrm{i}-2)} \ldots \mathrm{c}_{(\lambda 1-1) \mathrm{p}-(\lambda 1-2)} \\
& \mathrm{c}_{2} \mathrm{c}_{\mathrm{p}_{+1}} \ldots \mathrm{c}_{\left(\mathrm{i}_{1-1)} \mathrm{p}-\left(\mathrm{i}_{-3)}\right)\right.} \ldots \mathrm{c}_{(\lambda 1-1) \mathrm{p}-\left(\lambda_{1}-3\right)} \\
& \text {. } . \text {. }
\end{aligned}
$$

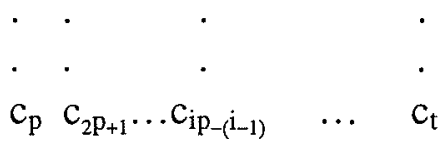

where $\left(c_{1}, c_{2}, \ldots, c_{t}\right)=$

$$
\begin{array}{ll}
(1, t, 2, t-1,3, \ldots, t / 2,(t+2) / 2) & \text { if } \mathrm{t} \text { is even } \\
(1, t, 3, t-2,5, \ldots,(t+1) / 2,(t+5) / 2, \ldots, t, 1) & \text { if } t=4 \gamma+1 \\
(1, t, 3, t-2,5, \ldots,(t-1) / 2,(t+3) / 2, \ldots, t, 1) & \text { if } t=4 \gamma+3
\end{array}
$$

The set $U_{i=2}^{t}\left(c_{i}-c_{i-1}\right)$ contains each non-zero number mod $t$ and so each pair of distinct treatments will appear once in the final design.

Example 4.6. Let $t=10$ and $p=4$. Then the above procedure produces the following column: $(1,10,2,9,3,8,4,7,5,6)$ and the design is presented below.

Now, let $\mathrm{t}=9$ and $\mathrm{p}=5$. Then the above procedure produces the following column: $(1,9,3,7,5,7,3,9,1)$ and the design is given below. 


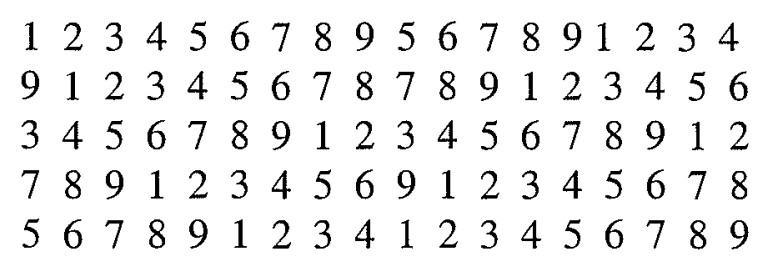

The following construction method due to Afsarinejad (1983) establishes the existence of strongly balanced minimal RMDs whenever $\mathrm{p}<\mathrm{t}$.

Construction 4.6. The construction method is the same as Construction (3.5) with the exception that the column is:

$\left(\mathrm{c}_{1}, \mathrm{c}_{2}, \ldots, \mathrm{c}_{\mathrm{t}}, \mathrm{c}_{\mathrm{t}+1}\right)=$

$\begin{array}{ll}(1, t, 2, t-1, \ldots, t / 2,(t+2) / 2,(t+2) / 2) & \text { if } \mathrm{t} \text { is even; } \\ (1, t, 3, t-2, \ldots,(t+5) / 2,(t+1) / 2,(t+1) / 2, \ldots, t, 1) & \text { if } t=4 \gamma+1 \\ (1, t, 3, t-2, \ldots,(t-1) / 2,(t+3) / 2,(t+3) / 2, \ldots, t, 1) & \text { if } t=4 \gamma+3\end{array}$

The set $U_{2}{ }^{t+1}\left(c_{i}-c_{i-1}\right)$ contains each number mod $t$ and so each ordered pair of treatments will appear precisely once in the final design.

Example 4.7. Let $t=10$ and $p=6$. Then, $\left(c_{1}, c_{2}, \ldots, c_{11}\right)=(1,10,2,9,3,8,4,7,5,6,6)$ and the deign obtained is presented below.

$\begin{array}{llllllllllllllllllll}1 & 2 & 3 & 4 & 5 & 6 & 7 & 8 & 9 & 10 & 8 & 9 & 10 & 1 & 2 & 3 & 4 & 5 & 6 & 7 \\ 10 & 1 & 2 & 3 & 4 & 5 & 6 & 7 & 8 & 9 & 4 & 5 & 6 & 7 & 8 & 9 & 10 & 1 & 2 & 3 \\ 2 & 3 & 4 & 5 & 6 & 7 & 8 & 9 & 10 & 1 & 7 & 8 & 9 & 10 & 1 & 2 & 3 & 4 & 5 & 6 \\ 9 & 10 & 1 & 2 & 3 & 4 & 5 & 6 & 7 & 8 & 5 & 6 & 7 & 8 & 9 & 10 & 1 & 2 & 3 & 4 \\ 3 & 4 & 5 & 6 & 7 & 8 & 9 & 10 & 1 & 2 & 6 & 7 & 8 & 9 & 10 & 1 & 2 & 3 & 4 & 5 \\ 8 & 9 & 10 & 1 & 2 & 3 & 4 & 5 & 6 & 7 & 6 & 7 & 8 & 9 & 10 & 1 & 2 & 3 & 4 & 5\end{array}$

Now, let $\mathrm{t}=9$ and $\mathrm{p}=4$. Then

$\left(c_{1}, c_{2}, \ldots, c_{10}\right)=(1,9,3,7,5,5,7,3,9,1)$ and the design is given below.

12234566788978891223456678991233456

$\begin{array}{lllllllllllllllllllllllllll}9 & 1 & 2 & 3 & 4 & 5 & 6 & 7 & 8 & 5 & 6 & 7 & 8 & 9 & 1 & 2 & 3 & 4 & 3 & 4 & 5 & 6 & 7 & 8 & 9 & 1 & 2\end{array}$

$\begin{array}{lllllllllllllllllllllllllll}3 & 4 & 5 & 6 & 7 & 8 & 9 & 1 & 2 & 5 & 6 & 7 & 8 & 9 & 1 & 2 & 3 & 4 & 9 & 1 & 2 & 3 & 4 & 5 & 6 & 7 & 8\end{array}$

$\begin{array}{lllllllllllllllllllllllllll}7 & 8 & 9 & 1 & 2 & 3 & 4 & 5 & 6 & 7 & 8 & 9 & 1 & 2 & 3 & 4 & 5 & 6 & 1 & 2 & 3 & 4 & 5 & 6 & 7 & 8 & 9\end{array}$

Again, these designs are not trend-free with respect to residual effects. Designs, which are trend-free with respect to both treatment effects and residual effects, exist and they are called circular balanced RMDs.

Fortunately, circular balanced RMDs can be constructed using nearest neighbour designs. The construction of these designs was given by Rees (1967) and has been considered by several authors, for example, Hwang (1973), Hwang and Lin (1974, 1976, 1977, 1978), Lawless (1971) and Street (1982), Street and Street (1987, chapter 14). We 
shall present a construction method and for the rest of methods we refer the interested readers to the above mentioned authors.

All it is needed is to construct an initial block to satisfy the following conditions, (I) all the differences, forward and backward, must be distinct; ( II ) the sum of the forward differences must be zero $(\bmod t)$. Then use this initial block and develop a design cyclically. Now find the mirror image of this initial block and develop another design cyclically. These two designs together constitute a circular balanced RMD.

Construction 4.7. Given $t=4 \gamma+3$ and $p=2 \gamma+1$ circular RMDs exists whenever $t$ is a prime power. Let $\mathrm{x}$ be a primitive root then the initial block for the first part of deign would be : $\left(x^{0}, x^{2}, x^{4}, \ldots, x^{4 r}\right)$ and the initial block for the second part of design would be $\left(x^{4 v}, \ldots, x^{4}, x^{2}, x^{0}\right)$.

Example 4.7. Let $t=7$ and $p=3$. Then initial block of the first part is. $(1,2,4)$ and the initial block of second part is $(4,2,1)$. The design is:

$\begin{array}{llllllllllllll}1 & 2 & 3 & 4 & 5 & 6 & 7 & 4 & 5 & 6 & 7 & 1 & 2 & 3 \\ 2 & 3 & 4 & 5 & 6 & 7 & 1 & 2 & 3 & 4 & 5 & 6 & 7 & 1 \\ 4 & 5 & 6 & 7 & 1 & 2 & 3 & 1 & 2 & 3 & 4 & 5 & 6 & 7\end{array}$

\section{ACKNOWLEDGEMENT}

I would like to thank Professor Paul Seeger for reading an earlier version of this paper and providing me with some very useful suggestions and comments. 


\section{REFERENCES}

Afsarinejad, K. (1983). Balanced repeated measurements designs. Biometrika 70, 563-568.

Afsarinejad, K. (1990). Repeated measurements designs-A review. Commun. Statist. Theory Meth., 19(11), 3985-4028.

Atkinson, A.C. and Donev A.N. (1996). Experimental designs optimally balanced for trend. Technometrics 38, 333-341.

Box, G.E.P. (1952). Multi-factor designs of first order. Biometrika 39, 49-57.

Box, G.E.P. and Hay, W.A. (1953). A statistical design for the removal of trends occurring in a comparative experiment with an application in biological assay. Biometrics 9, 304319.

Bailey, R.A., Cheng, C.S. and Kipnis, P. (1992). Construction of trend-resistant factorial designs. Statistica Sinica 2, 393-411.

Bradley, R.A., Yeh, C.M. (1980). Trend-free block designs: Theory. Ann. Statist. 8, 883893.

Bradley, R.A. and Odeh, R.E.(1988). A generating algorithm for linear trend-free and nearly linear trend-free block designs. Commun. Statist. -Simula. 17, 1259-1280.

Chai, F.S. and Majumdar, D. (1993). On the Yeh-Bradley conjecture on linear trend-free block designs. Ann. Statist. 21, 2087-2097.

Cheng, C.S. (1985). Run order of factorial designs. In proceedings of the Berekeley conference in honor of Jerzy Neyman and Jack Kiefer (vol. 2), eds. L.M. Lecam and R.A. Olshen, Belmont, CA: Wadsworth, 619-633.

Cheng, C.S. and Jacroux, M. (1988). The construction of trend-free run orders of two-level factorial designs. American Statistical Association 83, 1152-1158.

Coster, D.C. (1993). Trend-free run orders of mixed-level fractional factorial designs. Ann. Statist. 21, 2072-2086.

Coster, D.C. and Cheng, C.S. (1988). Minimum cost trend-free run orders of fractional factorial designs. Ann. Statist. 16, 1188-1205.

Cox, D.R. (1951). Some systematic experimental designs. Biometrika 38, 312-323.

Cox, D.R. (1952). Some recent work on systematic experimental designs. J. Roy. Statist. Soc. Ser. B 14, 211-219.

Cox, D.R. ( 1958). The planning of experiments. Wiley. New York. 
Daniel, C. (1976). Applications of statistics to industrial experimentation. Wiley, New York.

Daniel, C. and Wilcoxon, F. (1966). Factorial $2^{\mathrm{p}-\mathrm{q}}$ plans robust against linear and quadratic trends. Technometrics 8, 259-278.

Githinji, F. and Jacroux, M. (1998). On the determination and construction of optimal designs for comparing a set of test treatments with a set of controls in presence of a linear trend. J. Statist. Plann. Inference 66, 161-174.

Hill, H.M. (1960). Experimental designs to adjust for time trends. Technometrics 2, 67-82.

Hwang, F.K. (1973). Constructions for some classes of neighbour designs. Ann. Statist. 1, 786-790.

Hwang, F.K. and Lin, S. (1974). A direct method to construct triple systems. J. Combinatorial Theory. A 17, 84-94.

Hwang, F.K. and Lin, S. (1976). Construction of 2-balanced (n, k, $\lambda$ ) arrays. Pacific J. Maths. 64, 437-453.

Hwang, F.K. and Lin, S. (1977). Neighbour designs. J. Combinatorial Theory A 23, 303313.

Hwang, F.K. and Lin, S. (1978). Distributions of integers into k-tuples with prescribed conditions. J. Combinatorial Theory A 25, 105-116.

Jacroux, M. (1993). On the construction of trend-resistant designs for comparing a set of test treatments with a set of controls. American Statistical Association 88, 1398-1403.

Jacroux, M. (1994). On the construction of trend resistant mixed level factorial run orders. Ann. Statist. 22, 904-916.

Jacroux, M. and Ray, R.S. (1990). On the construction of trend-free run orders of treatments. Biometrika 77, 187-191.

Joiner,B.L. and Campbell, C. (1976). Designing experiments when run order is important. Technometrics 18, 249-259.

Kunert, J. (1985). Optimal repeated measurements designs for correlated observations and analysis by weighted least squares. Biometrika 72, 375-389.

Lawless,J.F. (1971). A note on certain types of BIBD's balanced for residual effects. Ann. Math. Statist. 42, 1439-1441.

Lin, M. and Dean, A.M. (1991). Trend-free block designs for varietal and factorial experiments. Ann. Statist. 19, 1582-1596. 
Mukerjee, R. and Sengupta, S. (1994). A-optimal run orders with a linear trend. Austral. J. Statist. 36, 115-122.

Ogilvie, J.C. (1963). A simple method for the elimination of individual trends in the analysis of balanced sets of Latin squares. Biometrics 19, 264-272.

Phillips, J.P.N.( 1964). The use of magic squares for balancing and assessing order effects in some analysis of variance designs. Appl. Statist. 13, 67-73.

Phillips, J.P.N. (1968a). A simple method of constructing certain magic rectangles of even order. Math. Gazette 52, 9-12.

Phillips, J.P.N. (1968b). Methods of constructing one-way and factorial designs balanced for trend. Appl. Statist. 17, 162-170.

Prescott, R.J. (1981). The comparison of success rates in cross-over trials in the presence of an order effects. Appl. Statist. 30, 9-15.

Rees, D.H. (1967). Some designs of use in serology. Biometrics, 23, 779-791.

Street, A.P. (1982). A survey of neighbour designs. Congressus Numerantium 34, 119-155.

Street, A.P. and street, D.J. (1987). Combinatorics of experimental design. Clarendon Press, Oxford.

Stufken, J. (1988). On the existence of linear trend-free block designs. Commun. Statist. Meth. 17, 3857-3863.

Taylor, J. (1967). The value of orthogonal polynomials in the analysis of changeover trials with dairy cows. Biometrics $23,297-311$.

Yeh, C.M. and Bradley, R.A. (1983). Trend-free block designs: existence and construction results. Commun. Statist. -Theor. Meth. 12, 1-24.

Yeh, C.M., Bradley, R.A. and Notz, W.I. (1985). Nearly trend-free block designs. American Statistical Association 80, 985-992.

Whittinghill, D.C. (1989). A note on trend-free block designs. Commun. Statist. Theory Meth. 18, 277-285.

Wikie,D. (1987). Analysis of factorial experiments and least squares polynomial fitting by the method of orthogonal polynomials for any spacing of the level of the independent variables. J. Appl. Statist. 14, 83-89. 


1999:1 Andersson, E.:

1999:2 Wessman, P.:

1999:3 Andersson, E.:

1999:4 Andersson, E.:

1999.5 Mantalos. P. \& Shukur, G.:

1999:6 Shukur, G.:

1999:7 Järpe, E. \& Wessman, P.:

1999:8 Johnsson, T.:
On monotonicity and early warnings with applications in economics.

The surveillance of several processes with different change points.

Monotonicity aspects on seasonal adjustment.

Monotonicity restrictions used in a system of early warnings applied to monthly economic data.

Testing for cointegrating relations- A bootstrap approach.

The effect of non-normal error terms on the properties of systemwise RESET test.

Some power aspects of methods for detecting different shifts in the mean.

On statistics and scientific thinking. 